\title{
Du musical au paysage sonore : en haine de l'harmonie?
}

\section{Baptiste Bacot}

\section{(2) OpenEdition}

12 Journals

Édition électronique

URL : http://journals.openedition.org/recherchestravaux/459

DOI : 10.4000/recherchestravaux.459

ISSN : 1969-6434

Éditeur

UGA Éditions/Université Grenoble Alpes

\section{Édition imprimée}

Date de publication : 15 mai 2011

Pagination : 169-184

ISBN : 978-2-84310-200-4

ISSN : 0151-1874

Référence électronique

Baptiste Bacot, « Du musical au paysage sonore : en haine de l'harmonie ? », Recherches \& Travaux [En ligne], 78 | 2011, mis en ligne le 15 novembre 2012, consulté le 08 septembre 2020. URL : http:// journals.openedition.org/recherchestravaux/459; DOI : https://doi.org/10.4000/recherchestravaux. 459 


\section{Du musical au paysage sonore : en haine de l'harmonie?}

Le titre de cet article relève à la fois de la surprise et de la provocation. Comment peut-on haïr l' "harmonie» - et que faut-il entendre par ce dernier terme qui prend tantôt un sens technique, tantôt un sens très général? Si l'on définit l'harmonie comme combinaison de hauteurs sonores dans le cadre de la tonalité, force est de reconnaître que les années d'après-guerre multiplient les discours hostiles à l'égard d'un système compositionnel que Pierre Boulez juge désormais «inutile». Issu du dodécaphonisme viennois, le sérialisme des années 50 a d'abord travaillé à partir des hauteurs avant de s'intéresser à l'ensemble des paramètres compositionnels (durée, timbre, attaque, etc.) et de remettre ainsi doublement en cause l'hégémonie du système tonal. Parallèlement à cet effort destiné à renouveler la création occidentale, les «musiques électroniques» commencent à se développer en Europe, proposant une autre voie d'émancipation à l'égard de la sacro-sainte tradition. Mais une fois encore, peut-on parler de «haine» envers l'harmonie? Si la formulation paraît sans doute bien excessive - on parlera plutôt de souci de la différence ou de l'indifférence -, il ne faut pas négliger que les «musiques électroniques» n'ont pas échappé à plusieurs formes de polémiques. Quand elles sont liées aux manifestations les plus diffusées de la culture populaire ou de la culture de masse, les musiques électroniques participent bon gré mal gré à la guerre symbolique qui oppose les différentes classes sociales ou générationnelles. Se donnant pour mission d'ébranler l'Europe aux anciens parapets ou diabolisées comme une opération de destruction de la culture classique (et de l'harmonie!), les "musiques électroniques" sont souvent prises entre deux feux, objets des discours les plus fantasmatiques. En partant de la haine, comme réalité ou comme représentation, cet article se donne 
pour ambition d'établir un état des lieux, préalable indispensable à toutes formes de discussion.

Aborder les "musiques électroniques" revient à se situer devant un champ dont l'étendue est aujourd'hui aussi vaste que l'est celle des ensembles «musiques traditionnelles» ou "musiques classiques» au sens large de ce terme. Trois tendances majeures au moins peuvent être recensées au sein des musiques électroniques. On les distinguera après un rappel historique visant à ouvrir des perspectives théoriques dans ce champ. Nous commencerons par situer historiquement le point de départ des musiques électroniques dans la pratique savante de la musique et présenter sa tripartition technique (bandes magnétiques, modules analogiques et données numériques). Les contours de trois pratiques compositionnelles distinctes en seront dégagés; l'une d'elle, ayant une position floue et entretenant des points de contact avec les deux autres, pourtant closes sur elles-mêmes, sera prise pour objet, ce qui, à notre connaissance, n'arrive que trop rarement ${ }^{\mathrm{t}}$. Une étude de cas viendra clore ce propos en engageant les fondements d'une réflexion liant linguistique, musicologique et esthétique. Ce travail lie ainsi théorie musicale et pratiques compositionnelles. Il détaille les implications esthétiques des technologies dont dépendent directement ces pratiques.

\section{Situations}

Certains artistes de la première moitié $\mathrm{du} \mathrm{xx}^{\mathrm{e}}$ siècle ont, avant même que les moyens techniques ne le permettent, l'intuition d'une musique qui n'est plus nécessairement jouée avec des instruments traditionnels. Deux démarches nous semblent illustrer ceci avec force. D'abord, celle du peintre futuriste Luigi Russolo, qui écrit en I9I3 dans L'Art des bruits :

Il faut élargir et enrichir de plus en plus le domaine des sons. Ceci répond à un besoin de notre sensibilité. Nous remarquons en effet que tous les compositeurs de génie contemporains tendent vers les dissonances les plus compliquées. En s'éloignant du son pur, ils arrivent presque au son-bruit. Ce besoin et cette tendance ne pourront être complètement satisfaits que par la jonction et la substitution des bruits aux sons ${ }^{2}$.

Ce premier point de conclusion du "manifeste futuriste» appelle plusieurs remarques. En premier lieu, l'import du bruit dans la musique est le résultat

I. Il est possible que l'absence de théories s'y rattachant directement telles qu'on les trouve dans la musique savante soit la raison de ce délaissement de ces musiques.

2. L. Russolo, L'Art des bruits [1913], traduction française par N. Sparta, Lausanne, L'Âge d'homme, coll. «Avant-gardes», 1975, p. 4I. 
d'un constat sur l'état du monde : l'industrialisation de l'occident au cours du XIX ${ }^{\mathrm{e}}$ siècle, qui va croissant au début du suivant (« les tintamarres différents des gares, des forges, des filatures, des imprimeries, des usines électriques et des chemins de fer souterrain $\left.s^{3} »\right)$, amène Russolo à prendre acte de la richesse des mondes sonores créés par les machines, et en comparaison, de la fadeur de l'orchestre. C'est en deuxième lieu une raison dictée par le plaisir esthétique, "un besoin de notre sensibilité», qu'invoque Russolo : "L'oreille d'un homme du XVIII ${ }^{e}$ siècle n'aurait jamais supporté l'intensité discordante de certains accords produits par nos orchestres [...]; Notre oreille au contraire s'en réjouit, habituée qu'elle est par la vie moderne, riche en bruits de toute sorte 4 ." On trouve enfin dans ce propos une formulation explicite du travail des "compositeurs de génie contemporains" qui, par leur approche de l'orchestration, tendent à dégager des masses sonores qui ne sont plus convoquées par l'harmonie mais par leur timbre intrinsèque. On pense bien sûr immédiatement au Sacre du printemps de Stravinsky (créé deux mois après que Russolo eut écrit L'Art des bruits), en particulier au deuxième mouvement de la première partie, "Augures printaniers" et à son accord martelé aux cordes et renforcé sur les accents par les cors, qu'il convient de considérer «dans sa spécificité, comme valeur sonore ${ }^{5}$ et non dans sa dimension harmonique fortement polysémique, et par conséquent inopérante. On pense aussi et surtout à Edgar Varèse qui n'aura cessé d'attendre que des «machines à sons» voient le jour afin de donner un élan décisif à la musique, à en croire ces mots :

Les avantages que je prévois sont ceux-ci : une machine semblable nous libérerait du système arbitraire et paralysant de l'octave; elle permettrait l'obtention d'un nombre illimité de fréquences, la subdivision de l'octave, et, par conséquent, la formation de toute gamme désirée; une étendue insoupçonnée de registres, de nouvelles splendeurs harmoniques que l'usage de combinaisons sub-harmoniques rendrait possibles; des sons combinés, des différenciations de timbres, des intensités inhabituelles au-delà de tout ce que peuvent accomplir nos orchestres; une projection du son dans l'espace par son émission de l'une ou l'autre partie d'une salle de concert, selon les besoins de l'œuvre; des rythmes qui s'entrecroiseraient indépendamment les uns des autres, simultanément en contrepoint, pour nous servir d'un mot ancien, puisque cette invention pourrait jouer toutes les notes voulues... ou fractions de notes dans une unité de temps ou de mesure donnée, tel qu'il est maintenant humainement impossible de le faire ${ }^{6}$.

3. Ibid., p. 38 .

4. Ibid., p. 36-37.

5. A. Boucourechliev, Igor Stravinsky, Fayard, coll. "Les indispensables de la musique», I982, p. 90.

6. Extrait d'une conférence donnée en 1939 à l'université de Californie du Sud, cité par O. Vivier, Varèse, Seuil, coll. «Solfèges», 1973, p. I25. 
À défaut d'avoir une telle machine en main - même s'il adjoindra les bandes magnétiques en stéréophonie à l'orchestre dans Déserts en I954 et les utilisera exclusivement dans Poème électronique, diffusé dans le Pavillon Philips de Xenakis et Le Corbusier dans le cadre de l'Exposition universelle de 1958 à Bruxelles - Varèse en imagine les grands principes. Cette réflexion trouve un écho dans ce qu'il conviendrait d'appeler les «tentatives orchestrales» du compositeur, par exemple dans Arcana (1927) où les masses de timbres (plus que de hauteurs) s'opposent et se répondent, ou dans Ionisation (I929-1932), qui ne mobilise que des instruments à percussions, le piano n'étant plus utilisé pour sa polyphonie, mais bien plutôt pour son "hyperphonie ${ }^{7}$ ", si l'on peut dire. Le vocabulaire de l'affranchissement domine dans les paroles de Varèse, laissant entrevoir un possible compositionnel inexploré. Ce qui est réellement en jeu ici, d'un point de vue historique, c'est, bien plus qu'une rénovation formelle (au sens musicologique de la structure d'une œuvre), une approche de la musique qui pense sous un jour nouveau ses premiers principes, ou, pour le dire de manière atténuée, qui déplace les préoccupations majeures du compositeur. Il n'est en effet plus question de l'organisation des hauteurs telle qu'elle se pose comme problème aux viennois durant la première moitié du $\mathrm{Xx}^{\mathrm{e}}$ siècle, par exemple. La proposition de Varèse semble bien plus vaste, impliquant tout à la fois rythme, timbre, dynamique, hauteur et, pour la première fois comme paramètre compositionnel, l'espace, régis ensemble par cette seule "machine à son ", et non plus par les seuls paramètres classiques de positionnement de l'orchestre. L'aspect coercitif de l'orchestre au moment où parle Varèse est à nouveau mis en exergue selon l'opposition son (musical) bruit. Chez Russolo comme chez Varèse, c'est cette opposition qu'il convient de fondre en une unité.

En 1948, les recherches électroacoustiques de Pierre Schaeffer aux studios de l'ORTF le conduisent lui aussi à substituer à la notion restreinte de «musical» celle, plus large, de «sonore», concrétisant ainsi les intuitions décrites précédemment, au moins en partie. Les bandes magnétiques (réduites dans un premier temps à des "études» de bruits, coups d'essai de la manipulation de ce matériau) invitent les compositeurs à travailler pour la première fois avec des outils qui ne sont pas des instruments mais des dispositifs reproduisant les sons. En effet, le son figé sur bandes peut provenir de n'importe quelle source sonore à la simple condition qu'elle puisse être enregistrée : instruments de l'orchestre, voix, mais aussi tous types de bruits urbains ou naturels. À ces nouveaux outils il faut ajouter le magnétophone (massivement utilisé par

7. Seuls des clusters sont joués par le piano, c'est-à-dire en définitive des sons inharmoniques. C'est la trop grande densité harmonique de ces sons qui rend l'oreille inapte à les réduire sous une fréquence fondamentale unique. 
les nazis pour retransmettre par la radio les harangues d'Hitler et récupéré par la société américaine Ampex qui sort en 1948 l'Ampex Model $200^{8}$ et popularisera l'appareil), mais aussi les dispositifs de traitement qui accompagnent les bandes magnétiques : filtres, grâce auxquels on peut isoler une ou plusieurs bandes de fréquences d'un enregistrement, réverbérations artificielles simulant l'espace, procédés de lectures décalées en cascade pour obtenir des effets de retard, chambres d'écho, lectures inversées, ralenties, accélérées, superposées, etc. Ceux-ci contribuent à l'abolition de la frontière, devenue de plus en plus ténue, entre son musical et bruit.

Peu de temps après, à partir de 1953, Stockhausen, de retour des studios de l'ORTF, explore aux studios de la Westdeutsche Rundfunk (WDR - créé entre autres par Herbert Eimert) à Cologne un pan qui complète les démarches de Pierre Schaeffer, celui de la musique électronique. Ce ne sont pas les bandes magnétiques qui servent de support au son, mais plus directement des générateurs d'ondes qui en sont la source (des sinusoïdes dans un premier temps, puis des formes d'ondes plus complexes). D'un côté du Rhin, les sons sont reproduits, et de l'autre, générés. Les traitements possibles du matériau premier, les ondes, sont d'ailleurs cependant généralement les mêmes que ceux que l'on trouve en France pour les bandes magnétiques.

L'étude "réfléchissante», pour parler en termes kantiens, de la structure physique du son - par le biais de spectrogrammes et d'analyseurs de fréquences - qui part du phénomène sensible pour en dégager une loi n'est rendue possible que par l'informatique. L'analyse et la synthèse numérique sont découvertes à travers les expérimentations de Matthews, Pierce et Guttman, travaillant avec des ordinateurs à la Bull Telephone Company en I959 et à la mise au point, cruciale pour cette démarche, du convertisseur analogique-numérique et numérique-analogique, permettant le codage d'un signal électrique en données numériques et réciproquement. Il devient alors possible de spécifier physiquement les sons avec une précision plus grande encore que celle des oscillateurs utilisés en Allemagne. On peut désormais avoir un contrôle encore accru sur la forme d'onde, reproduire à l'infini des types de sons définis par des algorithmes que l'on peut sauvegarder. La plus grande différence entre une approche analogique (avec des appareils physiques) et une approche numérique (avec des données) de la musique électronique est que dans le second cas, les écrans d'ordinateur livrent une représentation visuelle du son, ce qui permet une souplesse bien plus grande dans l'usage des traitements fréquentiel et spatial entre autres. Non seulement

8. Voir à ce sujet G. Kosmicki, Musiques électroniques, des avant-gardes aux dance floors, Marseille, Le Mot et le reste, coll. «Formes», 2009, p. 40. 
la forme d'onde fondamentale, générant un son, mais aussi les enveloppes ${ }^{9}$ ou les spectres ${ }^{\mathrm{IO}}$ permettent d'arriver rapidement à des imitations, plus ou moins satisfaisantes, d'instruments réels. C'est la méthode dite d' "analyse par synthèse». Cette approche timbrale de la structure physique du son permet parallèlement d'éclairer le comportement de l'oreille au contact de phénomènes sonores de différentes natures. Ainsi, dans Suite for Little boy (1968, en référence au nom donnée à la bombe qui ravagea Hiroshima en I945 et pour les décennies suivantes), Jean-Claude Risset utilise-t-il des «sons paradoxaux" (aussi nommés "trompe-oreille», filant ainsi la métaphore entre corps sonores et corps lumineux $\left.{ }^{\mathrm{II}}\right)$ : par manipulation du paramètre de la phase et par une réinjection constante d'harmoniques, notre oreille perçoit un son qui descend perpétuellement. Dans la seconde moitié de Mutations (1969), c'est une montée infinie qui est perçue. La spatialisation du son est enfin explorée: tout comme une manipulation peut donner corps à des "sons paradoxaux" mettant en jeu la hauteur, il est possible de créer des illusions spatiales, la stéréophonie prenant de plus en plus d'importance dans les années 60. Parallèlement à la stricte émulation d'instruments et de la recherche acoustique, les compositeurs (qui sont également chercheurs, on l'aura compris ${ }^{12}$ ), dégagent les frontières de mondes sonores inédits déployés par l'informatique, toujours susceptibles d'être déterminés avec une grande précision par la structure physique même du son que l'on entend produire.

En seulement vingt ans de recherches musicologiques, le progrès technique, réclamé par les compositeurs et développé par eux, a permis de prendre conscience du fait que la matière sonore ne se structure pas seulement en terme de hauteurs et de rythmes, mais qu'il est également possible de la sculpter ou de la ciseler en son timbre, et ce avant même de se préoccuper de son organisation relative. Pour le dire autrement, c'est l'organisation interne du son qui pose problème: des studios de Radio France aux laboratoires de la Bull Telephone Company, le son est d'abord étudié en tant que phénomène (représentation sensible), en tant qu'il prend vie dans l'oreille, tandis qu'il

9. Les paramètres d'attaque, de déclin, de soutien et de relâchement, du volume d'abord, et plus tard également des paramètres de filtres ou des oscillateurs secondaires qui font partie du processus de génération du son.

IO. C'est-à-dire la quantité et la qualité des harmoniques pour chaque bande de fréquence donnée.

II. «Le son est la lumière sous une autre forme... En musique, les instruments font l'office des couleurs qu'emploie le peintre" écrit Balzac dans la nouvelle "Gambara» (I839). Cité par J.-Cl. Risset, «Timbre» dans J.-J. Nattiez (dir.), Musiques. Une encyclopédie pour le XXI siècle, t. II, "Les savoirs musicaux», Arles, Actes Sud-Cité de la musique, 2004, p. I38.

I2. "L'exploitation des ressources du son numérique est [...] inséparable d'une recherche sur le son musical et sa perception.» écrit Risset lui-même dans l'article "Timbre» cité ci-dessus, p. I54. 
était auparavant abordé en grande majorité sous le problème de l'organisation des hauteurs les unes par rapport aux autres (autrement dit, en termes abstraits), le timbre étant considéré, selon Risset, comme un "paramètre résiduel $^{13}$ ». Dès lors, au cours des années 70 et 80 la lutherie électrique (guitare, basse, orgue Hammond et autres claviers électriques, ainsi que les pédales d'effets qui les accompagnent et que s'approprieront à des fins différentes les musiciens de rock et de jazz) gagne du terrain, avant de devenir électronique (synthétiseurs, samplers, boîtes à rythmes).

On peut alors distinguer nettement, à partir de la même trajectoire historique (et a fortiori du progrès technique), deux catégories dans les musiques électroniques. La première reste attachée à une tradition savante et à des perspectives de recherches en acoustique dont les œuvres lient compte rendu scientifique et forme artistique : Répons (198I-I983) de Pierre Boulez a valeur de démonstration technologique en mobilisant la machine $4 \mathrm{X}$, un processeur numérique de traitement acoustique en temps réel élaboré à l'IRCAM ( R» pour «recherche»). Ce mouvement peut toutefois être estimé pour ses qualités esthétiques propres (que nous comprenons comme un plaisir sensible et intellectuel). Cela n'est pas le cas de l'autre mouvement, qui donne naissance à une musique standardisée, sans réelle saveur intrinsèque, une musique qui ne propose pas, évaluée surtout selon des critères de quantité (d'écoute, de vente, de diffusion) : la musique populaire électrique et électronique, dont les morceaux persistent à employer des formes musicales toujours plaquées de l'extérieur et des règles d'harmonie tonale (niant donc le primat du timbre permis par les technologies mobilisées). La seule positivité de cette dernière réside dans sa propension à faire danser.

\section{Passerelle}

Néanmoins, en marge de ces deux tendances majeures closes sur elles, de nombreux groupes, mouvements ou musiciens désirent tirer profit - pas seulement financier - de ces possibles musicaux offerts par la technologie, sans pour autant avoir été formés à l'ingénierie informatique, au traitement du signal ou à la recherche musicologique. Ces artistes font un usage expérimental du son sans toutefois théoriser leur démarche, et cherchent à s'émanciper des formes connues - bref, ils partent du phénomène sonore pour arriver à la musique, là où d'autres en restent au donné. C'est, nous semble-t-il, dans cet entre-deux, ou dans ce ni-l'un-ni-l'autre que réside un vivier mal

13. Ibid., p. I34. 
connu de démarches musicales prolifiques, étant donné la démocratisation $\mathrm{du}$ home studio ${ }^{\mathrm{I4}}$. Comme un monde dans un monde, on y trouve des "compositeurs", au sens aussi noble que celui qu'on lui prête dans la musique savante : ceux-ci utilisent entre autres les outils informatiques élaborés dans des centres de recherches musicales et comptent parmi leurs influences (explicitement ${ }^{\text {t5 }}$ ou stylistiquement) certaines figures phares de la musique savante. Leurs créations nécessitent bien souvent plusieurs écoutes pour en saisir la dynamique. Dans la compilation An Anthology of noise and electronic music, certaines pièces de Varèse, Cage, Pousseur ou Xenakis côtoient des morceaux de Sonic Youth, Otomo Yoshihide, Einstürzende Neubauten, Autechre, ou DJ Spooky That Subliminal Kid. On regroupe ces derniers sous l'étiquette fourre-tout de "musique expérimentale». On y trouve également, frôlant cette fois la musique populaire par la simplicité schématique des structures et de l'harmonie, des "producteurs", ceux qui, tirant parti de la démocratisation des technologies musicales, réalisent des morceaux (et non plus des œuvres) destinés à la diffusion lors de concerts, par l'intermédiaire des disques-jockeys (DJ). Ceux-ci élaborent un mix en diffusant sans interruption des morceaux sur une platine vinyle, $\mathrm{CD}$ voire $\mathrm{MP}_{3}$, qui nécessite dans ce cas un ordinateur. On pourra questionner au moins trois aspects de ces mixes ou $D J$ sets. Il faut d'abord considérer l'aspect perlocutoire de ces sets, car le DJ s'adresse avant tout aux corps. On peut dire, pour justifier le recours à un terme linguistique, que le DJ peut prévoir, dans une certaine mesure, l'effet que le morceau (track) diffusé aura sur le public puisqu'il en connaît la structure et la teneur en termes de timbre et de fréquences. Par exemple, lors de ces soirées durant lesquels plusieurs DJ peuvent se succéder et diffusent ainsi des morceaux pendant plusieurs heures d'affilée, on peut observer de manière notable une réaction du public lorsqu'un morceau est nettement plus violent (ce caractère étant explicable par la prééminence de certaines fréquences ${ }^{16}$ ) ou bien que le tempo

I4. Équipement dont le centre est souvent l'ordinateur et autour duquel gravitent divers périphériques matériel ou logiciel comme des synthétiseurs ou des effets. Du fait de son faible coût, on peut avoir un home studio sans pour autant être professionnel.

I5. Parmi les influences recensées sur la page myspace de l'artiste Murcof (Fernando Corona), on trouve entre autres Giacinto Scelsi, Giya Kancheli ou Morton Feldman. Sa musique fait un usage massif de samples orchestraux qui pourraient être tirés d'œuvres d'Arvo Pärt et conserve un esprit minimaliste.

I6. Typiquement les basses fréquences $(20-200 \mathrm{~Hz})$ qui, à très fort volume traversent littéralement le corps - se munir de protections auditives lors de telles manifestations est indispensable. Ces pratiques musicales invitent à substituer la notion de "physio-acoustique ", engageant le corps entier, à celle de psycho-acoustique, qui nous semble ici dépassée. On peut par exemple faire mention des concerts de Sunn O))), «dont le volume sonore poussé à l'extrême provoque des vomissements au sein de l'audience», Y. Citton, «Le Percept noise comme registre du sensible", Multitudes n² 28, Amsterdam, printemps 2007, p. 138. 
gagne d'un seul coup cinquante numéros de métronome : les mouvements se font plus brusques et les cris laissent penser à une extériorisation d'un plaisir d'ordre esthétique trouvé dans ces moments. Par le choix des morceaux et leur enchaînement, le DJ réactualise une fonction chamanique de la musique ${ }^{17}$ et joue avec les réactions du public, créant l'attente, la comblant ou la décevant. Il y aurait également beaucoup à dire sur le statut ontologique de ces morceaux. Le DJ peut en effet planifier ses diffusions lors du concert à l'avance, ou bien puiser au fur et à mesure du set dans sa bibliothèque de vinyles (ou de $\mathrm{CD}$ ) et, d'une certaine façon, «improviser » son mix. Les morceaux au sein d'un mix sont donc mobiles. Par ailleurs, rares sont les DJ qui choisissent de diffuser un morceau dans son intégralité, ceux-ci étant coupés ou élidés en fonction des besoins du set. Enfin et surtout, les périodes de transition entre deux morceaux peuvent donner lieu à de véritables créations. À l'aide d'un égaliseur ${ }^{18}$, le DJ peut par exemple superposer la ligne de basse du morceau qui vient d'être diffusé à un élément différent du morceau suivant ${ }^{19}$. Il est dès lors possible d'amorcer au cours de cette transition des éléments d'un morceau qui sera diffusé bien plus tard - ou pas du tout. Cela pose donc problème : dans quelle mesure peut-on dire qu'un morceau susceptible d'être élidé, superposé, fragmenté ou traité existe au sein d'un mix? Et, d'une manière plus générale, que fait un DJ qui mixe, que devient la valeur de l'enregistrement dans le cadre de telles pratiques?

Nous nous sommes contentés jusqu'à présent d'amorcer des pistes de réflexion au sein du champ des musiques électroniques, dont on peut à présent donner une définition : la musique électronique est un type musical différent de la musique acoustique ou électrique (amplifiée). Elle se définit par l'utilisation essentielle de matériel électrique, électronique, ou numérique dans la production du matériau sonore. Elle mobilise surtout trois procédés : le sampling (ou échantillonnage : l'évolution des bandes magnétiques de la musique concrète), le traitement du signal audio et la synthèse sonore. Il est notable que l'interface physique de production du signal sonore est l'enceinte.

17. C'est-à-dire une musique qui, par ses structures, répétées, peut conduire à une mise à distance du corps, à son autonomisation et à se réceptivité esthétique propre. Ce point ne saurait d'ailleurs être tranché en une phrase.

I8. Un type de filtre à partir duquel on peut ajouter ou retirer jusqu'à I2 ou $24 \mathrm{~dB}$ sur quatre à huit points du spectre avec une largeur de bande - appelée «facteur Q" pour "qualité» - réglable.

19. C'est-à-dire la partie qui assume au plus haut point la fonction rythmique du morceau, générée à partir de boîtes à rythmes qui sont les avatars analogique ou numérique du batteur. 
Je voudrais maintenant, en guise de geste pratique, proposer une réflexion plus détaillée sur le premier des procédés qui rentre dans notre définition des musiques électroniques : le sampling, dont on peut déjà dire qu'il est essentiellement $\mathrm{cut}^{20}$.

\section{Le sample : étude de cas}

Je choisis de prendre pour objet lors de cette écoute analytique, qui éclairera par ailleurs certains procédés évoqués plus haut, un morceau du groupe lyonnais High Tone, Echo-logik, figurant sur l'album Bass température paru en 200 I chez Jarring Effects. Autour de l'analyse, opérée selon l'ordre temporel ${ }^{21}$, se tisse une réflexion sur les enjeux de la relation sample-musique. En un filigrane restreint, l'on esquisse les contours d'une analogie entre les structures stylistiques et les formes musicales induites par le sample.

Le morceau s'ouvre sur un sample de chant indien accompagné d'une danse (on entend le bruit des pieds au sol), extrait du film Little Big man (1970) d'Arthur Penn, à la suite duquel une voix d'homme éraillée énonce (source identique) :

Avance et viens te battre... C'est un bon jour pour mourir... Merci de m'avoir fait un être humain... Merci de m’avoir aidé à devenir un guerrier... Merci pour mes victoires, et pour mes défaites... Merci de m’avoir donné la vue... Et de m’avoir rendu aveugle pour que je voie plus loin... Tu fais toutes les créatures et tu les guides dans leur chemin, [pauvre père?]... Et maintenant, tu dois faire retourner les êtres humains au silence (pause)... Nous marcherons bientôt sur une route (pause)... Qui ne conduit nulle part.

Dès «mourir", la section rythmique se met en place : la basse, sourde, tout en croches, pose des repères harmoniques ( $f a$, note pôle du chant indien, et la bémol) tandis que le batteur égrène les croches sur la cymbale charleston. Rapidement, un coup de grosse caisse martèle le temps tandis que sur les temps 2 et 4 s'installe immuablement un coup de caisse claire en cross-stick (au lieu d'une frappe classique, la baguette repose sur la peau en dépassant du fût; elle est légèrement soulevée tout en gardant contact avec le centre de la peau et vient frapper le cercle en métal de la caisse claire, ce qui donne ce son sec, caractéristique du jeu des batteurs de reggae et de dub, également apprécié par les batteurs de jazz) sur laquelle on entend une réverbération dont la longueur peut varier selon les coups.

20. Nous empruntons cette idée selon laquelle le sample est avant tout «double coupure» le délimitant à B. Gallet dans Le Boucher du Prince Wen-Houei, Paris, Musica Falsa, 2002, p. I03.

2I. Nous renvoyons en note un minutage pour chaque articulation clé du morceau. 
Après "défaites», le DJ (qui ne "mixe» pas comme on l'expliquait plus haut, mais qui "scratche", on parle alors plus spécifiquement de turntablism, même si ces catégories se retrouvent sous le terme plus général de "DJing») lance la lecture d'un vinyle et l'arrête aussitôt en coupant l'extrémité du sample, ce qui donne cet effet de glissement (deux croches), régulièrement repris par un effet de delay ${ }^{22}$ dont la fréquence est synchronisée sur le tempo, à la blanche, soit tous les deux temps. La basse s'ouvre et gagne en brillance à cet instant : le filtre qui lui était appliqué (toutes les fréquences supérieures à environ $\mathrm{I} 4 \mathrm{O} \mathrm{Hz}$ ne passent pas) est enlevé. Sur "plus loin», le DJ intervient à nouveau sur un motif rythmique (deux doubles croches - deux croches) plus complexe qui passe également dans un delay. La guitare entre et se superpose aux coups de caisse claire sur les temps pairs de la mesure en accords plaqués dans lesquels on retrouve les notes pôles du morceau. Un delay lui est également appliqué, mais à une fréquence très courte (autour de $80 \mathrm{~ms}$ ). Après «silence», le second motif rythmique du DJ refait progressivement surface sous une forme modifiée, plus étirée et lâche, certainement due au changement des paramètres du delay. Après "route", la section rythmique s'allège (plus de grosse caisse ni de caisse claire et la basse reprend son état fréquentiel initial) à l'exception de la guitare qui plaque maintenant ses accords tous les temps. Dans le même temps, le guitariste fait aller et venir le plateau de sa pédale wah-wah ${ }^{23}$. En même temps que "route», un sample est diffusé à deux reprises ("Yava»), noyé de réverbération et de delay. On le retrouve plus loin, en extension et dans sa version originelle car la hauteur est ici modifiée.

L'introduction prend fin sur un ultime motif de scratch et le morceau est lancée ${ }^{4}$. On retrouve la section rythmique (guitare, basse, batterie) sur laquelle se greffe un clavier qui déploie à nouveau un jeu typiquement reggae qui vient compléter la régularité de la guitare. Ce clavier, probablement un synthétiseur, a des caractéristiques timbrales bien précises : un filtre mouvant - dans l'esprit de la wah-wah - ouvre et ferme en alternance le son de l'instrument, dont l'oscillateur est par ailleurs modulé par un Low Frequency Oscillator (LFO) - à la différence d'un oscillateur générant directement un son, les très basses fréquences du LFO sont utilisées pour moduler le

22. Le delay est un effet qui répète pour un temps donné un signal qui lui est envoyé. On trouve toujours sur un delay, qu'il soit numérique ou analogique, les paramètres suivants : fréquence de la répétition (exprimée de la triple croche à la ronde d'un tempo donné ou bien en millisecondes) et le nombre de répétitions (appelée feedback et qui permet une lecture supplémentaire unique comme une boucle infinie).

23. Rendue populaire par Jimi Hendrix, elle n'est en réalité qu'un filtre qu'on actionne au pied. Lorsque le plateau est complètement poussé vers l'extérieur, côté orteils, toutes les fréquences passent, et lorsqu'il est au point le plus bas, côté talon, le son est très étouffé. 24. I'38". 
paramètre désiré). À la fin de cette première carrure, tout s'arrête et ne persistent que les sons de la cymbale charleston et d'un accord de guitare qui passent dans une chambre d'écho, utilisée notamment à la WDR. La différence notable entre l'écho et le delay est que le premier «dégrade» le son au fur et à mesure des répétitions en grignotant progressivement le bas du spectre, ce que ne fait pas le second. La section rythmique reprend son chemin sans le clavier. Le batteur enrichit subtilement son jeu en décalant les accents de caisse claire et le bassiste descend la gamme de $f a$ mineur à partir de la quinte do en fin de phrase. Le sample de voix disant «lookin' for honey» est alors diffusé trois fois par le DJ (on le reconnaît au crépitement caractéristique du diamant dans le sillon du vinyle). La syllabe «loo» de «lookin'» et la syllabe «ney» de "honey» sont situées, en termes de hauteur, à une grande proximité $\mathrm{du} f a$, la tonique du morceau. On peut donc supposer, du point de vue de la composition, que le sample a été déterminé avant le choix de la tonalité du morceau : il devient le primat de la musique et impose ses lois internes aux hauteurs. Lui succède alors un sample de voix identique énonçant quelque chose comme "David honey boney", puis un troisième, d'une voix différente cette fois, disant "[incompréhensible] haven't fight for your musical rights». Cette carrure est répétée, enrichie par le clavier qui refait son apparition et, sur les derniers mots du troisième sample, le roulement de caisse claire (cette fois avec une frappe classique, l'olive attaquant directement la peau, et non un rimshot) passe dans un delay dont la fréquence augmente alors même que l'effet est appliqué, ce qui provoque ce ralentissement. On peut penser à bon droit que cette manipulation est effectuée en studio, et par un des techniciens son lors de concerts.

Le passage suivant $\mathrm{t}^{25}$ se caractérise par des accords de premier degré et de degré $\mathrm{V}$ ( $\mathrm{fa} 5$ / do 7 sans sensible) en alternance, tenus au clavier et toujours plaqués sur les temps pairs pour la guitare. Le sample amorcé à la fin de l'introduction est déployé dans sa totalité : «Yava [?] honey moon». Il se présente comme une réponse sémantique aux premières phrases par la reprise du terme "honey" et comme une dominante pour "lookin' for honey", puisque chaque syllabe possède une hauteur (respectivement la bémol et do pour "Yava» et si bémol, la bémol et si bémol pour "honey moon»). Le sample est diffusé trois fois d'affilée sous trois formes différentes : "Yava honey moon", puis "Yava» seul (avec delay), et enfin "Yava [?] honey moon... he said [?]" (avec delay persistant sur le dernier mot). Le degré $V$ est tenu par le duo guitare clavier en manière de demi-cadence.

25. 2' 46 '. 
Les samples suivants, diffusés par le DJ (les craquements s'entendent très bien dans ce passage) "Are you ready?" et "Yes, of course» (avec delay) mobilisent comme l'équivalent de ce que Jakobson appelle la fonction phatique du langage, interpellant l'auditeur pour maintenir le contact avec lui en l'exhortant à prêter attention à ce que suit. C'est bien ici une fonction narrative du sample qui est à l'œuvre, annonçant un moment nouveau du récit musical mis en place par les musiciens. À l'attention à la musique se superpose donc l'attention à un discours. Il semblerait que l'on puisse établir une analogie entre les structures du récit et les structures formelles de la musique du fait du sens - en ses deux acceptions : comme porteur sémantique et comme direction musicale - induit par les samples ${ }^{26}$. Les deux samples sont énoncés par deux voix différentes : une voix de femme pour le premier, et une voix d'enfant pour le second. On ne peut en déduire quoi que ce soit par rapport au contexte originel de ces voix (probablement tirées elles aussi d'un film) : elles peuvent effectivement être telles qu'elles sont présentées dans ce morceau, une question et sa réponse, ou bien peuvent être issues de deux contextes absolument distincts. Quoi qu'il en soit, elles semblent être en adéquation l'une avec l'autre dans le contexte précis du morceau. Quel est donc l'élément auquel l'auditeur doit prêter attention? C'est un coup de caisse claire en cross-stick ${ }^{27}$ extrait à l'aide d'un sampler au cours du morceau puis réinjecté à la fréquence du sextolet et qui occupe une bonne partie de la carrure suivante. Cette répétition à outrance, qui fait perdre de vue la source originale et donne accès à une autre dimension de ce son instrumental est sculptée à l'aide d'un filtre passe-bas (qui laisse passer les basses fréquences et coupe les hautes fréquences) et de son paramètre de résonance (qui accentue les fréquences autour desquelles le filtre agit). Par ailleurs, ce sample lu en boucle se déplace dans l'espace stéréophonique, donnant l'impression d'échapper au contrôle des musiciens.

Après une nouvelle apparition du sample "Yava honey moon he...", seule la section rythmique au complet reprend son chemin habituel. La basse est alourdie par une légère distorsion dont l'intensité augmente progressivement. Lorsqu' elle semble avoir atteint son apogée dans l'épaisseur, le batteur signale par quatre coups de grosse caisse l'arrivée d'un élément nouveau : un solo de

26. Ceux-ci peuvent en effet avoir un rôle descriptif comme dans l'introduction qui plante un paysage musical aride emprunt de sacré, comme le rôle d'embrayeurs de discours comme "Are you ready?». Dans cette perspective appelant une réflexion plus vaste, il nous semble qu'une analyse des musiques utilisant des samples puisse être éclairée par les outils de la grammaire de discours et des schémas communicationnels. 27. 3'18". 
scratch $^{28}$. Le DJ utilise un sample très court extrait du début de Sex machine de James Brown (dont les parties instrumentales et les gimmicks vocaux été abondamment échantillonnés, notamment par les musiciens de hip hop dans les années 80) : pour lancer la musique, le chanteur de funk dit «one, two, three, four". Le dernier mot, "four» est exclu de ce sample pour donner "one, two, three», sur lequel scratche le DJ. Ce fragment très court est travaillé, probablement de manière improvisée, par le DJ qui ne dispose que de deux couples de possibilités : avancer (lire) - reculer (lire de manière rétrograde) tout en dosant la vitesse de lecture et ouvrir - fermer le passage pour le signal audio (volume) au moyen d'un bouton coulissant que l'on déplace latéralement (crossfader). Jouant sur ces deux paramètres combinés (sens de lecture et volume), le sample est diffusé un grand nombre de fois d'affilée durant cette période, mais on n'entend en réalité qu'un fragment de cet échantillon à chaque fois. C'est en quelque sorte une atomisation du matériau initial qui donne lieu à une reconstruction informelle ${ }^{29}$ douée d'une valeur propre. Un autre sample est ensuite éprouvé, celui d'un bruit de dérapage ou de pneus qui crissent sur l'asphalte (qu'on ne reconnait jamais comme tel). Lors des deux derniers mouvements de la main du DJ qui manie le vinyle sur la platine, on entend distinctement la lecture presque entière du sample original et la lecture inversée. Après une dernière carrure qui prend appui sur Yava honey moon, le morceau s'achève dans les répétitions du delay.

Cette analyse aura permis de montrer d'abord que dans la musique électronique, le sample a un rôle compositionnel bien plus important que l'harmonie elle-même. C'est à partir des caractéristiques intrinsèques d'un sample que s'élabore la musique : dans le cas de ce morceau, l'harmonie est déduite des intonations de voix. Il ne s'agit donc en aucun cas d'un simple collage comme on aurait pu le craindre, puisque le sample, sans affecter la musique de l'extérieur, entretient une relation d'interdépendance avec elle. L'usage de samples permet en outre de dynamiser la structure musicale, qu'il sera dès lors aisé de relier à des catégories issues de la théorie littéraire, notamment en termes narratologique et linguistique. Chaque partie s'articule autour d'un ou plusieurs samples précis dont l'autonomie (contexte original) disparait dans l'unité du morceau, ou, pour le dire autrement, le sample et les instruments s'amalgament et fusionnent. Le sampling donne lieu à de nouveaux gestes musicaux dont la déformation, voire l'informe est au fondement, tant dans le scratch que dans l'usage du sampler lui-même.

28. 4'09".

29. Cette reconstruction est qualifiée d' "informelle» car lorsqu'elle est donnée à entendre, il est impossible de dégager l'unité interne, les structures du sample: la fragmentation l'a rendue méconnaissable. 
Alors, haine de la musique? Non, pas de la musique, indéterminée et vague, autrement dit de toutes les musiques. Les musiques électroniques ne haïssent pas la musique (ce serait absurde!) et n’hésitent pas à se nourrir des musiques déjà existantes, empruntées souvent à des civilisations lointaines. C'est souvent un hommage pouvant prendre la forme d'une citation instrumentale, comme c'est le cas dans de nombreux autres morceaux du groupe, dans lesquels des phrases d'instruments extraoccidentaux comme le shamisen (Japon) ou le ney (Iran) font thème. Ceux-ci sont issus d'enregistrements préexistants, mais les membres du groupe, possédant eux-mêmes quelquesuns de ces instruments, tendent de plus en plus souvent à fabriquer leurs propres samples pour les besoins de leur musique. Alors, haine de l'harmonie, comme le titre le laissait entendre avec un sens évident de la provocation? Le recours à des modèles extraeuropéens participe incontestablement de cette volonté d'élargir les horizons et de s'éloigner du système tonal dont on éprouve parfois l'essoufflement. En même temps, comme on l'a vu, les musiques électroniques, relevant des formes les plus galvaudées de la culture de masse, n'hésitent pas à recourir aux enchaînements d'accords les plus élémentaires pour y plaquer sans risque-tout le strass d'une "orchestration" prétendument moderne. Porté par un souci d'émancipation beaucoup plus efficace, on insistera plutôt sur le rôle crucial de samples à caractère narratif tels qu'on les trouve dans l'analyse proposée. La musique se nourrit alors de formes littéraires ou cinématographiques qui lui assignent un dynamisme nouveau : la différenciation et la répétition sont alors supplantées par des schèmes issus des formes artistiques à récit qui rendent possibles des structures musicales immanentes, c'est-à-dire directement imputables au matériau lui-même.

Si elles semblent portées parfois par la haine (haine toute superficielle de la «musique classique» ou des concerts embourgeoisés), les musiques électroniques participent en profondeur au travail de rénovation des formes de la création. Bien sûr, ces musiques envahissent trop souvent notre paysage sonore pour ne pas susciter la haine de tous ceux qui entendent sans écouter, de tous les passants qui sont arrachés à leur musique intérieure par la tyrannie des décibels. Comme le rappelle Pascal Quignard, les oreilles n'ont pas de paupières - et, dans les rues de la ville, l'agression vient davantage des musiques électroniques les plus banales que des quatuors de Webern... Mais les musiques électroniques, qu'elles éprouvent ou suscitent épisodiquement la haine, valent beaucoup mieux que la réputation qu'on leur fait - cet article n'ayant pas d'autre ambition que de replacer un tel art au cour des musiques authentiquement contemporaines. 
À défaut de haine, reste pourtant une forme de violence... La puissance de la musique du groupe, dévoilée au plus haut point lors de concerts, a des propriétés cathartiques et on pourrait dire que la relation du groupe au public tient presque de l'affrontement, le premier ayant la volonté d'avoir un impact physique sur le second, qui résiste. La haine (mais s'agit-il de haine?) prend alors une forme ludique et abstraite (sans objet), médiatisée par la musique, et entre ainsi sur le terrain sociologique. 